Inhaled corticosteroids and decline in lung function in asthma

\section{Inhaled corticosteroids moderate lung function decline in adults with asthma}

\section{P Ernst}

\section{Further evidence of the benefit of long term inhaled corticosteroids in asthma}

s nhaled corticosteroids are the main stay of asthma treatment. They have earned this central place in our therapeutic armamentarium by their rapid alleviation of persistent asthma symptoms, improvement in lung function, prevention of exacerbations including those severe enough to require hospitalisation, and by preventing asthma deaths, even at low doses. ${ }^{12}$ They appear indicated as maintenance therapy for asthma patients who have more than occasional respiratory symptoms, ${ }^{3}$ and probably need to be used regularly to provide benefit. ${ }^{4} \mathrm{~A}$ recent study suggesting the contrary used a protocol of initial intense treatment with inhaled and oral corticosteroids which is not readily applicable clinically. ${ }^{5}$

Patients with asthma have a more rapid decline in lung function over time which results in significant irreversible airways obstruction in a substantial minority (estimates range from $23 \%$ to $33 \%) .^{6-9}$ Such fixed airflow limitation in asthma appears to be a manifestation of airway remodelling. ${ }^{10}$ While corticosteroids have many actions which might be expected to limit such remodelling, there is no consensus as to their efficacy in vivo. ${ }^{11}$ Several studies have suggested that the decline in lung function might be less in those making regular use of inhaled corticosteroids, but the number of subjects taking corticosteroids regularly has been small. ${ }^{12}{ }^{13}$ Furthermore, even among subjects with mild asthma inhaled corticosteroids appear to be limited in their capacity to prevent deterioration in lung function. ${ }^{14}$ The benefit of inhaled corticosteroids may be greater if used earlier in the course of the disease, ${ }^{15} 16$ although certain aspects of remodelling appear resistant to short courses of inhaled corticosteroids independent of the duration of symptoms. ${ }^{17}$ When beneficial effects of inhaled corticosteroids on remodelling have been observed, this has been achieved only after 12 months of treatment-much slower than their anti-inflammatory effect. $^{18}$

In this issue of Thorax Lange and colleagues, using information from the
Copenhagen City Heart Study, describe a reduction in the decline in forced expiratory volume in 1 second $\left(\mathrm{FEV}_{1}\right)$ over 10 years in patients with asthma aged over 30 years who were using inhaled corticosteroids throughout the observation period. ${ }^{19}$ Overall, inhaled corticosteroids reduced the annual decline in $\mathrm{FEV}_{1}$ by $23 \mathrm{ml} /$ year compared with asthma patients not using inhaled corticosteroids, and this benefit was of similar magnitude in smokers and nonsmokers. To put the size of this benefit into context, smoking over the 10 year observation period was associated with a $10 \mathrm{ml} /$ year faster decline in lung function.

There are several limitations to the study which are acknowledged by the authors. Firstly, asthma is defined by self-report only and the diagnosis of asthma might be more secure among those taking inhaled corticosteroids. The group not taking inhaled corticosteroids may contain more subjects with chronic obstructive pulmonary disease (COPD) who are well known to have rapid declines in $\mathrm{FEV}_{1} \cdot{ }^{20}$ Furthermore, those subjects taking inhaled corticosteroids might also be consistently more compliant with environmental measures which would contribute to the lesser decline in $\mathrm{FEV}_{1}$. Conversely, asthma patients who use inhaled corticosteroids might be expected to have more severe asthma and thus a greater decline in lung function. Such confounding by indication would cause an underestimation of the benefit of inhaled corticosteroids on decline in lung function in the current study. Finally, the choice of $\mathrm{FEV}_{1}$ as an index of remodelling and irreversible loss of lung function is not optimal. A better index would have been the post-bronchodilator $\mathrm{FEV}_{\mathrm{l}} / \mathrm{FVC}^{8}{ }^{8}$ Part of the decline in $\mathrm{FEV}_{1}$ observed in the study by Lange and colleagues may be related to ongoing poor asthma control rather than to irreversible airflow limitation

Even with these limitations, the study by Lange et al provides strong evidence that regular treatment with inhaled corticosteroids favourably alters the natural history of airway disease over the long term in patients with asthma. Stronger evidence is unlikely to be obtained, given the length of follow up required to be confident as to the significance of a reduction in $\mathrm{FEV}_{1}$ decline $^{21}$ and the ethical implications of withholding inhaled corticosteroids in a randomised trial.

While the benefit of inhaled corticosteroids on the decline in lung function was seen for the group as a whole, it is likely that the degree of benefit varied significantly between patients. The prognosis of asthma is highly variable, with many patients having consistently mild disease. ${ }^{22}$ For these patients, a small dose of inhaled corticosteroids can be expected to provide maximum benefit while, for others, fixed airflow limitation will develop with the usual approach to treatment. ${ }^{7}$ The AMPUL study group have already shown that remodelling may be decreased by intensifying asthma treatment in response to the degree of airway hyperresponsiveness, ${ }^{23}$ while examination of sputum allows us to identify patients less likely to respond to inhaled corticosteroids and for whom new approaches are needed. ${ }^{24}$

Thus, despite the further evidence of the benefit of inhaled corticosteroids in asthma provided by the study by Lange and colleagues, it behoves us to characterise our patients better in order to maximise the benefit of treatment in individual patients.

Thorax 2006;61:93-94

doi: 10.1136/thx.2005.042010

Correspondence to: Dr P Ernst, Division of Clinical Epidemiology, Royal Victoria Hospital, Montreal, Quebec, Canada, H3A 1A1; pierre. ernst@clinepi.mcgill.ca

Competing interests: Pierre Ernst has received speaker fees from and/or is on advisory boards of Altana, Astra Zeneca, GlaxoSmithKline, Merck, and Novartis.

\section{REFERENCES}

1 Barnes PJ. Inhaled glucocorticoids for asthma. N Engl J Med 1995;332:868-75.

2 Suissa S, Ernst P. Inhaled corticosteroids: impact on asthma morbidity and mortality. J Allergy Clin Immunol 2001;107:937-44.

3 Pauwels RA, Pedersen S, Busse WW, et al. Early intervention with budesonide in mild persistent asthma: a randomised, double-blind trial. Lancet 2003;361:1071-6.

4 Suissa S, Ernst P, Kezouh A. Regular use of inhaled corticosteroids and the long term prevention of hospitalisation for asthma. Thorax 2002;57:880-4

5 Boushey HA, Sorkness CA, King TS, et al. Daily versus as-needed corticosteroids for mild persistent asthma. N Engl J Med 2005;352:1519-28

6 Lange P, Parner J, Vestbo J, et al. A 15-year follow-up study of ventilatory function in adults with asthma. N Engl J Med 1998;339:1 194-200.

7 Ulrik CS, Backer V. Nonreversible airflow obstruction in life-long nonsmokers with moderate to severe asthma. Eur Respir J 1999;14:892-6. 
8 Rasmussen F, Taylor DR, Flannery EM, et al. Risk factors for airway remodelling in asthma manifested by a low post bronchodilator $\mathrm{FEV}_{1} /$ vital capacity ratio: a longitudinal population study from childhood to adulthood. Am J Respir study from childhood to adulthood.

9 Covar RA, Spahn JD, Murphy JR, et al. Progression of asthma measured by lung function in the childhood asthma management program. Am J Respir Crit Care Med 2004; 170:234-41.

10 Lazaar AL, Panettieri RA Jr. Is airway remodelling clinically relevant in asthma? Am J Med 2003;115:652-9.

11 Beckett PA, Howarth PH. Pharmacotherapy and airway remodelling in asthma? Thorax 2003:58: 163-74.

12 Grol MH, Gerritsen J, Vonk JM, et al. Risk factors for growth and decline of lung function in asthmatic individuals up to age 42 years. A 30 year follow-up study. Am J Respir Crit Care Med 1999: 160:1830-7.

13 Boulet LP, Jobin C, Milot J, et al. Five-year changes in airflow obstruction and airway responsiveness in mild to moderate asthma. Clin Invest Med 1994; 17:432-42.

14 Childhood Asthma Management Program Research Group. Long-term effects of budesonide or nedocromil in children with asthma. N Engl J Med 2000;343:1054-63.

15 Haahtela $T$, Jarvinen $M$, Kava $T$, et al. Effects of reducing or discontinuing inhaled budesonide in patients with mild asthma. N Engl J Med 1994;331:700-5.

16 Selroos O, Pietinalho $A$, Lofroos $A B$, et al. Effect of early vs late intervention with inhaled corticosteroids in asthma. Chest 1995; 108:1228-34.

17 Boulet LP, Turcotte H, Laviolette M, et al. Airway hyperresponsiveness, inflammation, and subepithelial collagen deposition in recently diagnosed versus long-standing mild asthma. Influence of inhaled corticosteroids. Am J Respir Crit Care Med 2000; 162:1308-13.

18 Ward C, Pais M, Bish R, et al. Airway inflammation, basement membrane thickening and bronchial hyperresponsiveness in asthma. Thorax 2002;57:309-16.
19 Lange $\mathbf{P}$, Ulrik CS, Scharling $\mathrm{H}$, et al. Inhaled corticosteroids and decline of lung function in community residents with asthma. Thorax 2006:61:100-4.

20 Fletcher C, Peto R. The natural history of chronic airflow obstruction. BMJ 1977;1:1645-8.

21 Dales RE, Hanley JA, Ernst $P$, et al. Computer modelling of measurement error in longitudinal lung function data. J Chron Dis 1987:8:769-73.

22 Ernst P, Cai B, Blais $L$, et al. The early course of newly diagnosed asthma. Am J Med 2002;112:44-8.

23 Sont JK, Willems LN, Bel EH, et al. Clinical control and histopathologic outcome of asthma when using airway hyperresponsiveness as an additional guide to long-term treatment. The AMPUL Study Group. Am J Respir Crit Care Med 1999:159:1043-51.

24 Green RH, Brightling CE, Woltmann G, et al Analysis of induced sputum in adults with asthma: identification of subgroup with isolated sputum neutrophilia and poor response to inhaled corticosteroids. Thorax 2002;57:875-9.

\section{Exercise induced bronchoconstriction in elite athletes: measuring the fall}

\section{K Holzer, J A Douglass}

\section{Decreased $\mathrm{FEV}_{1}$ in response to bronchial provocation challenge remains the method of choice for diagnosing EIB in elite athletes, but the exact level of fall which represents an abnormal response still needs to be determined}

$\mathrm{T}$ he problem of exercise induced asthma (EIA) in elite athletes was first recognised because of the disproportionately high numbers of athletes at the elite level using $\beta_{2}$ agonists for asthma treatment during competition. In response to this problem, the International Olympic Committee's Medical Commission (IOC-MC) and other major sporting bodies have instituted guidelines requiring objective evidence of asthma to permit medication use by elite athletes in competition. ${ }^{1}$ These guidelines have highlighted the need for bronchial provocation challenge tests in the diagnosis of asthma in the elite athlete group.

\section{Exercise induced bronchoconstriction (EIB)}

EIA describes the transitory increase in airway resistance that occurs following vigorous exercise. ${ }^{2}$ However, because exercise may be the only provoking factor in some patients, especially in elite athletes where there are no other symptoms or signs of asthma, the term "exercise induced bronchoconstriction" (EIB) may be more appropriate. This allows the separation of a physiological response from a disease. ${ }^{3}$

\section{Problems with diagnosing EIB}

Emerging data suggest that the clinical diagnosis of EIB is relatively inaccurate. Rundell and co-workers found that only $61 \%$ of athletes positive to a field exercise challenge test reported symptoms while $45 \%$ of those negative to the challenge also reported symptoms. ${ }^{4}$ Similar findings were reported in a study of 50 elite Australian summer sport athletes by Holzer and co-workers ${ }^{5}$ in which seven of the 26 athletes $(27 \%)$ with a positive challenge test for EIB reported no exercise related respiratory symptoms, and only 24 of the 34 athletes $(71 \%)$ who reported symptoms had a positive bronchial provocation challenge test. These findings indicate that some athletes who are clearly symptomatic after exercise and who may exhibit performance decrements may have normal spirometric values after exercise. The presence or absence of symptoms is therefore not a reliable indicator for diagnosing EIB.

\section{Available bronchial provocation challenge tests}

The diagnosis of EIB demands confirmation by demonstration of a decrement in lung function associated with exercise or a surrogate, usually achieved by bronchial provocation testing. There are a range of bronchial provocation challenge tests available to screen for or confirm the diagnosis of EIB, each with differing efficacy in the diagnosis as a consequence of the different methods and agonists used. Importantly, the efficacy of each of these challenge tests in the diagnosis of asthma in an individual with chronic asthma may be different from that in the diagnosis of EIB in athletes.

The eucapnic voluntary hyperventilation $(\mathrm{EVH})$ test is the current challenge test recommended by the IOC as the optimal laboratory based challenge test for the identification of EIB. ${ }^{1}$ Phillips et $a l^{6}$ showed that the airway response in asthmatics-as measured by changes in forced expiratory volume in l second $\left(\mathrm{FEV}_{1}\right)$ and specific conductance (sGaw)-to hyperpnoea with 5\% $\mathrm{CO}_{2}$ was similar to that provoked in the same asthmatic subjects by exercise at the same ventilation. The protocol used required the subject to perform hyperpnoea by inhaling dry air containing 5\% $\mathrm{CO}_{2}$ at room temperature for 6 minutes at a ventilation equivalent to 30 times baseline $\mathrm{FEV}_{1}$. EVH has been reported to have a high specificity for active asthma, diagnosing $90 \%$ of asthma cases when a fall in $\mathrm{FEV}_{1}$ of $10 \%$ is taken as abnormal and $100 \%$ when a $15 \%$ fall is considered abnormal. ${ }^{7}$ The symptoms provoked by $\mathrm{EVH}$ are very similar to those that occur following exercise (cough, chest tightness, dyspnoea, and wheeze). The major advantage of using EVH over exercise to provoke bronchoconstriction is the ability of the subject to reliably achieve and sustain a minute ventilation that is higher than that which could be obtained on exercise.

Pharmacological challenge tests, which rely on the administration of agents such as histamine and methacholine to act 